\title{
Detecting hepatic nodules and identifying feeding arteries of hepatocellular carcinoma: efficacy of cone-beam computed tomography in transcatheter arterial chemoembolization
}

\section{Yasuhiro Ushijima ${ }^{1}$, Tsuyoshi Tajima ${ }^{1,2}$, Akihiro Nishie ${ }^{1}$, Yoshiki Asayama ${ }^{1}$, Kousei Ishigami ${ }^{1}$, Masakazu Hirakawa ${ }^{1,3}$, Daisuke Kakihara' ${ }^{1}$, Daisuke Okamoto', Hiroshi Honda ${ }^{1}$}

${ }^{1}$ Departments of Clinical Radiology, Graduate School of Medical Sciences, Kyushu University, Fukuoka 812-8582, Japan.

${ }^{2}$ Department of Radiology, Graduate School of Medicine, Tokyo Women's Medical University, Tokyo 162-8666, Japan.

${ }^{3}$ Department of Radiology, Kyushu University Beppu Hospital, Oita 874-0838, Japan.

\begin{abstract}
Aim: To evaluate the effectiveness of using cone-beam computed tomography (CBCT) in transcatheter arterial chemoembolization (TACE) to detect hapatocellular carcinoma (HCC) nodules and their feeding arteries. Methods: Twenty-four patients with HCCs who underwent TACE using CBCT in addition to conventional digital subtraction angiography (DSA) were enrolled. After both conventional DSA and CBCT through the hepatic artery were acquired, TACE were performed. The nodules were defined as an HCC when dense accumulation of iodized oil was found within the nodule on CT obtained 2 weeks after the TACE. The number of detected nodules and identified feeding arteries, and their correlations with anatomical locations were assessed. Results: A total of $39 \mathrm{HCC}$ nodules (tumor diameter, 7-40 mm; mean, $17.4 \pm 7.9 \mathrm{~mm}$ ) were detected. Thirty-one nodules were detected by DSA alone but 8 nodules were additionally detected by adding CBCT to DSA. There were 53 feeding arteries associated with the $39 \mathrm{HCC}$ nodules. Among these arteries, 21 were identified by DSA alone; however, 47 were identified by combining CBCT with DSA. Additional feeding arteries, especially for the nodules located at the right and caudate lobes, were identified by CBCT. On the other hand, there was no difference in detection of nodules between the anatomical locations by CBCT. Conclusion: The use of CBCT in addition to DSA offers potential for increasing the number of detected nodules, and the number of their feeding arteries at the right and caudate lobes. CBCT might improve the quality of TACE procedure for HCC than DSA alone.
\end{abstract}

Key words: Hepatocellular carcinoma; transcatheter arterial chemoembolization; cone-beam computed tomography; interventional procedure

\section{Corresponding author:}

Dr. Akihiro Nishie, Department of Clinical Radiology, Graduate School of Medical Sciences, Kyushu University, Maidashi 3-1-1, Higashi-ku, Fukuoka 812-8582, Japan. E-mail: anishie@radiol.med.kyushu-u.ac.jp

\section{INTRODUCTION}

Transcatheter arterial chemoembolization (TACE), an endovascular intervention for hepatocellular carcinoma (HCC), is an established treatment procedure performed worldwide along with other therapeutic techniques such as surgical resection and percutaneous treatment. It has many advantages such as less invasiveness, the ability to act on

\begin{tabular}{|l|c|}
\hline \multicolumn{2}{|c|}{ Access this article online } \\
\hline \multirow{2}{*}{ Website: } & Quick Response Code \\
www.hrjournal.net & \\
DOI: & \\
10.20517/2394-5079.2016.32 & \\
\end{tabular}

multiple lesions, and the ability to be utilized on lesions in areas where percutaneous treatment is anatomically difficult to perform. In recent years, treatment with superselective catheterization has become easier, thanks to the advances in angiography devices such as the microcatheter and micro-guide wire. Recent TACE treatment results, such as

This is an open access article distributed under the terms of the Creative Commons Attribution-NonCommercial-ShareAlike 3.0 License, which allows others to remix, tweak, and build upon the worknon-commercially, as long as the author is credited and the new creations are licensed under the identical terms.

For reprints contact: service@oaepublish.com

How to cite this article: Ushijima $Y$, Tajima T, Nishie A, Asayama $\mathrm{Y}$, Ishigami K, Hirakawa M, Kakihara D, Okamoto D, Honda $\mathrm{H}$. Detecting hepatic nodules and identifying feeding arteries of hepatocellular carcinoma: efficacy of cone-beam computed tomography in transcatheter arterial chemoembolization. Hepatoma Res 2016;2:231-6. 
the local suppression rate and survival rate, are reported to have improved compared with previous results. ${ }^{[1-3]}$

Previously, endovascular intervention mainly employed conventional cut-film angiography; however, the usage of digital subtraction angiography (DSA) and the interventional radiology features (IVR)-CT system, combining conventional angiography with $\mathrm{CT}$, has now become more prevalent. The IVR-CT system can obtain tomographic images when performing IVR, providing much useful additional information. CT hepatic arteriography and CT arterioportography are recognized as techniques with the highest detection rates in HCC diagnosis. ${ }^{[47}$ However, because the IVR-CT system obtains information from two separate X-ray imaging devices (DSA and CT equipments), difficulties are often experienced while correlating the two sets of data obtained from the two devices.

Angiography devices equipped with a flat panel detectorbased cone-beam CT (CBCT) imaging system can provide three-dimensional and tomographic images on a single $\mathrm{X}$-ray device through rotational CBCT imaging in addition to a conventional two-dimensional DSA image. Furthermore, because of no change in the conventional angiography equipment, no additional space is required for the IVR-CT system. ${ }^{[8,9]}$

We hypothesized that a three-dimensional understanding of hepatic artery anatomy and multiple planar reconstruction (MPR) images obtained from arbitrary cross-section may contribute to nodule detection and feeding artery identification by combining CBCT imaging with conventional DSA imaging in endovascular intervention for HCC. In this study, we retrospectively examined the HCC nodule detection and feeding artery identification capabilities of CBCT imaging.

\section{METHODS}

\section{Subjects}

The subjects were 24 patients (12 males and 12 females) from our facility with clinically suspected HCC who underwent TACE using CBCT in addition to conventional digital subtraction angiography (DSA). The trial period was from October 2006 to January 2008. The patients were aged between 52 and 84 years (average age, 71.2 years). All patients had underlying chronic hepatitis or cirrhosis. In all cases, a dynamic study using multi-row detector computed tomography (MDCT; Aquilion-16 or Aquilion-64; Toshiba, Tokyo, Japan) was conducted within one month before TACE. All patients were clinically diagnosed with HCC by dynamic CT and/or the elevation of tumor markers.

\section{Imaging device}

The angiography equipment used in endovascular intervention was AXIOM Artis dBA (Siemens). In addition to conventional two-dimensional DSA imaging, rotation of the detector with the $\mathrm{C}$-arm helped in three-dimensional rotational imaging.
The protocol used for the three-dimensional rotating image was as follows: detector, $30 \mathrm{~cm} \times 38 \mathrm{~cm} \times 154 \mu \mathrm{m}$; FOV, LR $22.5 \mathrm{~cm} \times$ AP $22.5 \mathrm{~cm} \times$ HF $18 \mathrm{~cm}$; matrix, $1,024 \times$ 1,024; projection, 30 projection/s for $5 \mathrm{~s}$, rotation $200^{\circ}$; dosage, $1.2 \mu \mathrm{Gy} /$ pulse; contrast agent, iopamidol solution (150 mg l/mL) (Iopamiron 150; Bayer; Osaka, Japan); infusion rate and duration, $1-2.5 \mathrm{~mL} / \mathrm{s}, 8 \mathrm{~s}$; and delay time, $3 \mathrm{~s}$. Both upper limbs were raised, and imaging was performed with the patients holding their breath. The data obtained were transferred to an X-Leonard workstation (Siemens) and maximum intensity projection (MIP), volume-rendering (VR), and MPR (axial, coronal, and sagittal thickness, $3 \mathrm{~mm}$ ) images were generated.

\section{Endovascular intervention (TACE)}

First, a $3 \mathrm{~F}$ or $4 \mathrm{~F}$ sheath was inserted by the percutaneous approach from the groin into the femoral artery, and two-dimensional DSA imaging of the celiac and superior mesenteric arteries was performed using diagnostic catheters. Next, a two-dimensional DSA image of the hepatic artery (any one of the common, proper or replaced hepatic arteries) was obtained, and maintaining the catheter in the same location, CBCT three-dimensional rotating imaging was performed. As mentioned above, the CBCT volume data were processed in the workstation. Using this information, we performed superselective catheterization of the subsegmental branches of the hepatic artery. After confirming tumor staining on the two-dimensional DSA image, a suspension of the chemotherapeutic agent epirubicin (farmorubicin, 10-40 mg; Kyowa Hakko, Tokyo, Japan) and iodized oil (lipiodol, 1-6 mL; Andre Guerbet, Aulnay-sous-Bois, France) was infused arterially. Embolization was performed using a gelatin sponge (Gelpart, 1-10 mg; Nippon Kayaku/Astellas, Tokyo, Japan) in patients with preserved hepatic function (Child-Pugh classification A). In addition, for diagnostic purposes, small amounts of iodized oil were introduced into the subsegmental arterial branches, including those supplying the densely stained tumors. After two weeks of treatment, CT imaging was performed to confirm the presence or absence of iodized oil deposition.

\section{Evaluation}

HCC was defined as vascular enhancement on DSA imaging after superselective catheterization and nodular deposition of Lipiodol on CT imaging after treatment. Retrospectively, the study coordinator (Y.U.) reviewed all DSA, CBCT and CT imaging after treatment and recorded the size and location of each HCC on a subsegmental basis. The gold standard of a feeding artery was also based on tumor staining on DSA imaging after superselective catheterization. First, two radiologists engaged in interventional radiology (M.H. and D.K.) evaluated the presence of HCC on DSA from a common, proper or replaced hepatic artery with or without CBCT in a consensus fashion. When a focal vascular enhancement was seen, they diagnosed it as HCC. Next, the identification of a feeding artery was also attempted in subsegmental branch unit. A feeding artery was defined as a vessel continuing with tumor stain and visualized separately 


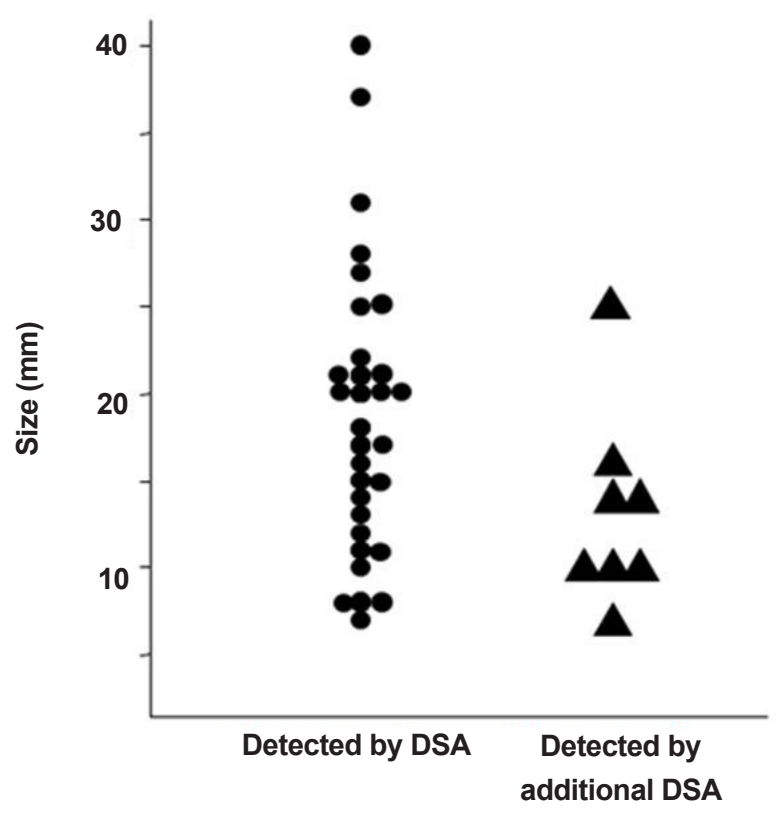

Figure 1: The correlation between detectoin of nodule and its size by DSA and additional CBCT. DSA: digital subtraction angiography; CBCT: cone-beam computed tomography

from other vessels. The number, size and location of HCC nodules detected, and the number and location of feeding arteries identified were compared between DSA images with and without CBCT. Comparison between mean sizes of nodules detected on DSA with and without CBCT was statistically performed using a Student's $t$-test. A $P$ value of $<0.05$ indicated a statistically significant difference.

\section{RESULTS}

A total of 39 HCCs were confirmed by the study coordinator. The size of these nodules on CT ranged from a diameter of 7-40 $\mathrm{mm}(17.4 \pm 7.9 \mathrm{~mm})$, and they were located at S1 $(n=5), \mathrm{S} 2(n=1), \mathrm{S} 3(n=3), \mathrm{S} 4$ $(n=5)$, S5 $(n=2)$, S6 $(n=5)$, S7 $(n=7)$, and S8 $(n$ $=11$ ). DSA imaging alone detected 31 nodules, but the additional eight nodules, which were difficult to identify with DSA imaging alone, were detected by combining DSA imaging with $\mathrm{CBCT}$ imaging. The diameter of the 31 nodules was $18.4 \pm 1.4 \mathrm{~mm}$ and that of the additional eight nodules was $13.3 \pm 2.3 \mathrm{~mm}$. The $P$-value of Student's $t$-test was 0.09 $(>0.05)$. No significant difference was observed between the two methods [Figure 1]. The sites of the detected nodules are displayed in Figure 2. A maximum of two extra nodules were detected in any given subsegment with the addition of CBCT imaging, and no bias by location was observed in nodule detection.

Fifty-three feeding arteries were associated with the 39 HCC nodules. Among them, 21 arteries were identified by DSA imaging (angiography from the proximal hepatic artery), however, 26 additional feeding arteries were identified by combined application of DSA and CBCT imaging. The relationship between feeding arteries and sites are displayed in Figure 3. Nodules with an additional number of feeding

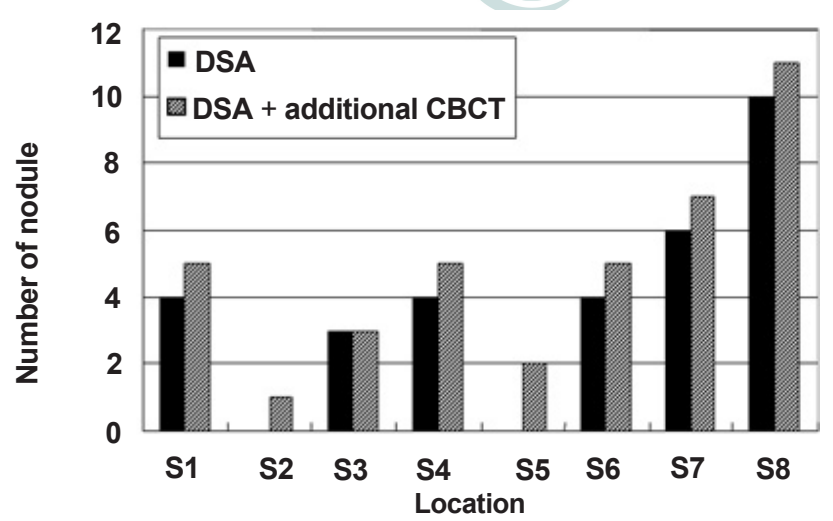

Figure 2: The correlation between detection of nodule and its location by DSA and DSA + CBCT. In any subsegments, extra nodules detected by additional CBBT were less than two. No bias by location was observed in nodule detection. DSA: digital subtraction angiography; $\mathrm{CBCT}$ : cone-beam computed tomography

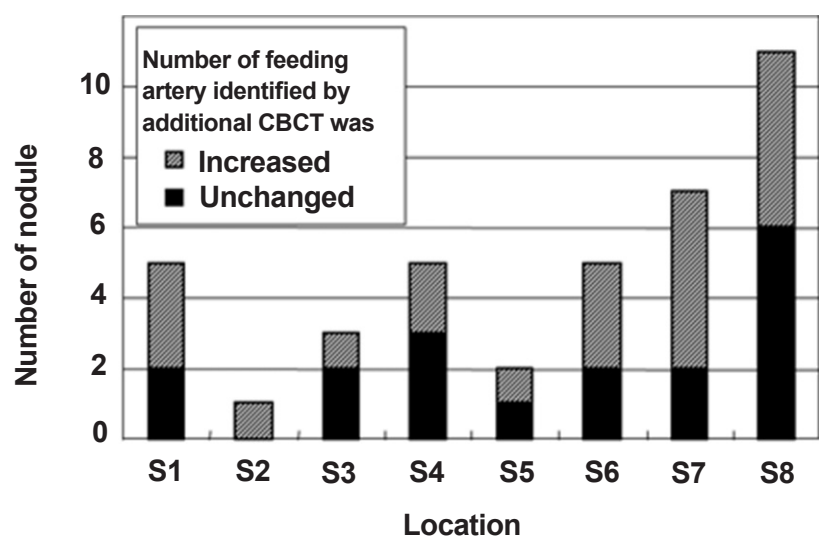

Figure 3: The correlation between identification of feeding artery and its location by additional CBCT. Number of nodules, in which number of their feeding arteries identified by additional CBCT was increased, was more than three in S1, S6, S7 and S8. On the other hand, the number of nodules was one or two in S2, S3, S4 and S5. $\mathrm{CBCT}$ : cone-beam computed tomography

arteries were observed in each subsegment, although an obvious increase in feeding arteries was observed in nodules located in the right and caudate hepatic lobes.

\section{DISCUSSION}

The CBCT imaging system is a device in which an X-ray radiation beam and a two-dimensional detector are rotated around the subject, and a three-dimensional image is reconstructed from the two-dimensional data. Because a two-dimensional detector is used, axial scanning of the body is not required. Thus, compared to images with conventional X-ray CT, images with a greater axial spatial resolution of the body can be acquired with $\mathrm{CBCT}$ imaging. Although the density resolution is inferior to CT, arbitrary tomographic images can be obtained in addition to the three-dimensional images. ${ }^{[10]}$ In recent years, various IVR procedures using these techniques were reported..$^{[11-14]}$ In this study, we examined the HCC detection and feeding artery identification capabilities of CBCT imaging in order to clarify its utility in endovascular intervention. 

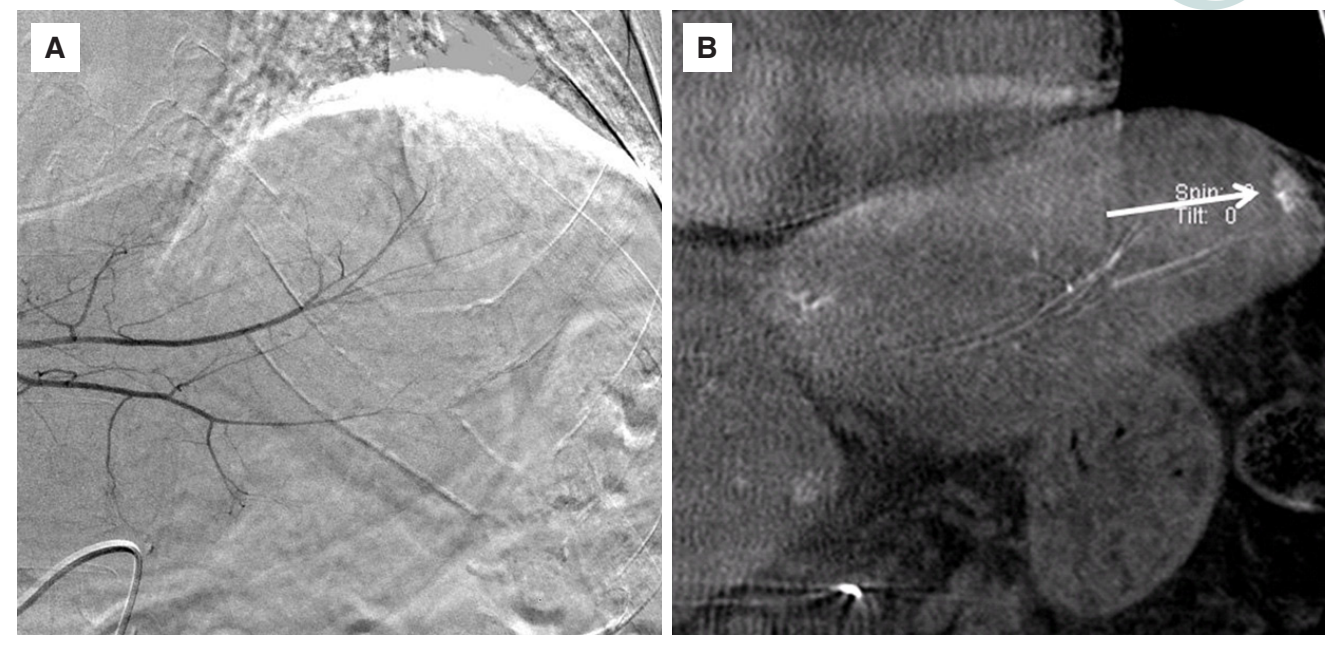

Figure 4: A 64-year-old man with hepatocellular carcinoma in the S2 of the liver. (A) Conventional digital subtraction angiography didn't show tumor stain, affected by heart pulsation; (B) coronal multiple planar reconstruction image of cone-beam computed tomography showed a hypervascular tumor with a diameter of $14 \mathrm{~mm}$ in the liver (arrow)

With regard to nodule detection ability, DSA imaging combined with CBCT imaging detected all 39 hepatic nodules, including the eight nodules that were difficult to identify with DSA imaging. Miyayama et al. ${ }^{[15]}$ previously reported that small HCC nodules approximately $1 \mathrm{~cm}$ in size, undetected by DSA imaging, were detected with CBCT imaging, thus making TACE possible for such cases. The average nodule diameter in our study was similar to that in the study by Miyayama et al. ${ }^{[15]}$ at $13 \mathrm{~mm}$. In contrast, Kakeda et al. ${ }^{[16]}$ reported that CBCT imaging did not detect tumors but detected feeding arteries. This is believed to be because their evaluation was based only on MIP and VR images, with small or weakly enhanced nodules not being revealed during three-dimensional reconstruction. In this study, evaluation was made using three-dimensional MPR images as well as MIP and VR images. Evaluation with MPR images is considered necessary for the identification of small nodules and nodules with weak enhancement. The advances in diagnostic imaging technology in recent years have made fine dynamic imaging with MDCT and liver-specific MRI contrast agents possible, and the small hepatic nodules identified by these methods are becoming increasingly subjective to endovascular intervention. ${ }^{\left[{ }^{17]}\right.}$ It is believed that CBCT imaging could become a useful tool for identification of such small nodules.

No bias by site was observed in nodule detection with $\mathrm{CBCT}$ imaging, although Figure 4 clearly shows that CBCT imaging is considered useful in areas where DSA imaging alone is insufficient because of cardiac pulsations and respiratory movements.

With regard to feeding artery identification capabilities, CBCT imaging was superior in identifying feeding arteries associated with nodules located in the right hepatic lobe. This lobe is deep anteroposteriorly, and the right anterior and posterior branches of the hepatic artery often overlap, leading to poor isolation in two-dimensional images of the anteroposterior direction. ${ }^{[18,19]}$ The addition of CBCT three-dimensional imaging (MIP and VR images) facilitates the easy isolation of anteroposterior overlapping vessels. A detailed search for feeding arteries associated with the nodules may be conducted if tomographic images (MPR) are used after understanding the general vessel anatomy with three-dimensional imaging [Figure 5]. Another advantage of $\mathrm{CBCT}$ imaging is that it helps in freely determining the most favorable working angle to isolate the feeding arteries and nodules that are the target of treatment, using three-dimensional images as a reference. This also makes three-dimensional CBCT images useful in superselective catheterization.

The number of feeding arteries identified by CBCT imaging in caudate lobe nodules has also increased. Endovascular intervention for HCC in the caudate lobe is generally considered technically difficult. ${ }^{|20|}$ Caudate branches are high in number and variation. They branch off from the proximal side of the right or left hepatic arterial trunks, as well as from the distal branches to the right lobe. ${ }^{[21,22]}$ These thin branches arising from thick vessels are difficult to identify with two-dimensional DSA imaging, further making selective catheterization difficult. In addition, multiple caudate branches become feeding arteries. With the addition of CBCT imaging in such cases, we can appreciate the detailed anatomical relationship between nodules and caudate branches with three-dimensional and tomographic imaging of the vessel, which is considered useful in selecting an appropriate working angle.

In endovascular intervention for HCC, we place a catheter in the common hepatic artery (proper or replaced hepatic artery) and obtain mapping CBCT images with the purpose of establishing therapeutic objectives. CBCT imaging with selective catheterization is believed to be useful in confirming the territory of the tumor that was stained before arterial infusion and embolization, ${ }^{[21]}$ although it is not routinely performed at our facility because its utility is unclear. In addition, frequent imaging takes time and effort. 

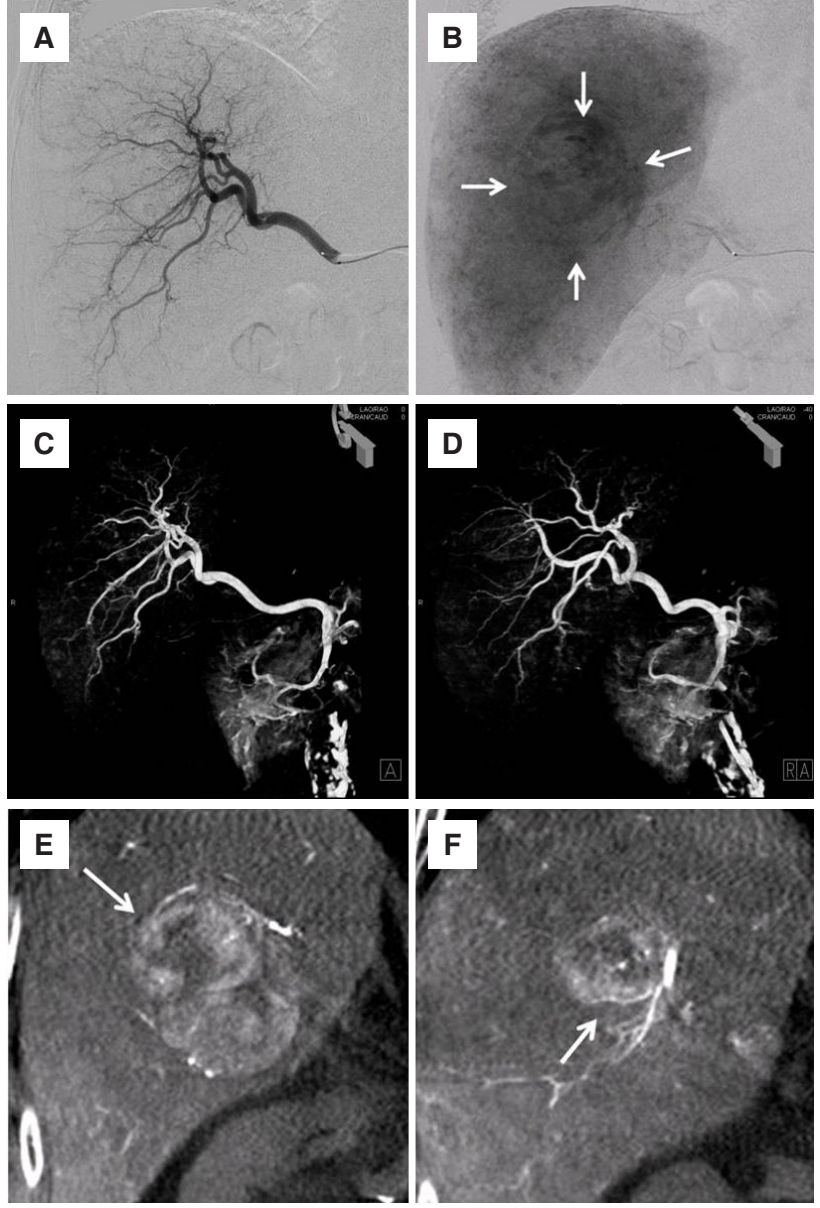

Figure 5: An 84-year-old man with hepatocellular carcinoma in the right lobe of the liver. Conventional digital subtraction angiography of the arterial phase $(A)$ and parenchymal phase (B) showed right hepatic artery branches and tumor stain with a diameter of $40 \mathrm{~mm}$ in the right lobe of the liver (arrow). Separation of the right hepatic artery branches was poor. It was difficult to identify the feeding vessel of the tumor. Volume-rendering image of the CBCT in frontal $(C)$ and right oblique (D) view obtained the three-dimensional information in the right hepatic artery and the branches could be separated. Coronal multiple planar reconstruction image of the $\mathrm{CBCT}(\mathrm{E}, \mathrm{F})$ showed the hypervascular tumor and $\mathrm{A} 7$ branches of the right hepatic artery (arrow) as feeding arteries. CBCT: cone-beam computed tomography

CBCT imaging is a technique that provides information useful in endovascular intervention for HCC, although there is adequate scope for debate regarding when and how it should be used. Many cases of endovascular intervention for HCC have been effectively performed solely based on conventional two-dimensional DSA imaging. In addition, the raising of both upper extremities, positioning, and reducing the concentration of contrast medium takes time when performing $\mathrm{CBCT}$ imaging, as does processing the threedimensional images. Even if CBCT imaging is performed, conventional two-dimensional DSA imaging cannot be completely excluded, with the amount of radiation exposure greater than conventional two-dimensional DSA imaging. When these factors are taken into consideration, performing routine $\mathrm{CBCT}$ imaging may not yet be suitable for all cases of HCC requiring endovascular intervention.
In our examination, there was increased identification of feeding arteries associated with HCC in the right and caudate hepatic lobes. CBCT imaging is also reported to be useful for detecting $\mathrm{HCC}$ of approximately $1 \mathrm{~cm}$ in size that were unidentified following DSA imaging. CBCT imaging is considered desirable in such cases.

Advances in technology will simplify CBCT imaging, reduce radiation exposure, and process images faster If all three-dimensional imaging becomes possible, then three-dimensional treatment using $\mathrm{CBCT}$ imaging is expected to become routine practice, replacing the traditional two-dimensional treatment procedure in endovascular intervention for HCC. ${ }^{[23]}$

There are some limitations in this study. First, the field of view by CBCT was narrower than DSA. Therefore, CBCT is unable to obtain a complete liver image in some cases. We should set the field of view around the region of interest in the liver. Next, we defined HCC as vascular enhancement on DSA imaging after superselective catheterization and nodular deposition of Lipiodol on CT imaging after treatment. There were no residual HCCs on CT performed two-weeks after treatment. However, we cannot exclude the possibility of very small residual HCCs. Moreover, we did not evaluate the specificity of detection of HCC nodules and their feeding arteries.

In this study, adding $\mathrm{CBCT}$ imaging to conventional twodimensional DSA imaging increased the HCC detection and feeding artery identification capabilities, especially in the right and caudate hepatic lobes. CBCT may be a useful, complementary modality for the endovascular intervention of HCC.

\section{Financial support and sponsorship} Nil.

\section{Conflicts of interest}

There are no conflicts of interest.

\section{Ethical approval}

This article does not contain any studies with human participants or animals performed by any of the authors. The retrospective design of the study was approved by the Institutional Ethics Committee and the requirement for informed written consent was waived.

\section{REFERENCES}

1. Miraglia R, Pietrosi G, Maruzzelli L, Petridis I, Caruso S, Marrone G, Mamone G, Vizzini G, Luca A, Gridelli B. Efficacy of transcatheter embolization/chemoembolization (TAE/TACE) for the treatment of single hepatocellular carcinoma. World J Gastroenterol 2007;13:2952-5.

2. Camma C, Schepis F, Orlando A, Albanese M, Shahied L, Trevisani F, Andreone P, Craxì A, Cottone M. Transarterial chemoembolization for unresectable hepatocellular carcinoma: meta-analysis of randomized controlled trials. Radiology 2002;224:47-54.

3. Llovet JM, Bruix J. Systematic review of randomized trials for unresectable hepatocellular carcinoma: chemoembolization improves 
survival. Hepatology 2003;37:429-42.

4. Nishie A, Yoshimitsu K, Asayama Y, Irie H, Tajima T, Hirakawa M, Ishigami K, Nakayama T, Kakihara D, Nishihara Y, Taketomi A, Honda H. Radiologic detectability of minute portal venous invasion in hepatocellular carcinoma. AJR Am J Roentgenol 2008;190:81-7.

5. Nishie A, Yoshimitsu K, Irie H, Tajima T, Hirakawa M, Ishigami K, Ushijima Y, Okamoto D, Nishihara Y, Taketomi A, Honda H. Radiological detectability of minute hepatic venous invasion in hepatocellular carcinoma. Eur J Radiol 2009;70:517-24.

6. Nishie A, Tajima T, Asayama Y, Ishigami K, Hirakawa M, Ushijima Y, Kakihara D, Okamoto D, Fujita N, Taketomi A, Yoshimitsu K, Honda H. Radiological assessment of hepatic vein invasion by hepatocellular carcinoma using combined computed tomography hepatic arteriography and computed tomography arterial portography. Jpn J Radiol 2010;28:414-22.

7. Murakami T, Oi H, Hori M, Kim T, Takahashi S, Tomoda K, Narumi Y, Nakamura H. Helical CT during arterial portography and hepatic arteriography for detecting hypervascular hepatocellular carcinoma. AJR Am J Roentgenol 1997;169:131-5.

8. Hirota S, Nakao N, Yamamoto S, Kobayashi K, Maeda H, Ishikura R, Miura K, Sakamoto K, Ueda K, Baba R Cone-beam CT with flat-paneldetector digital angiography system: early experience in abdominal interventional procedures. Cardiovasc Intervent Radiol 2006;29:1034-8.

9. Wallace MJ, Kuo MD, Glaiberman C, Binkert CA, Orth RC, Soulez G. Three-dimensional C-arm cone-beam CT: applications in the interventional suite. J Vasc Interv Radiol 2008;19:799-813.

10. Kalender WA, Kyriakou Y. Flat-detector computed tomography (FDCT). Eur Radiol 2007;17:2767-79.

11. Binkert CA, Alencar H, Singh J, Baum RA. Translumbar type II endoleak repair using angiographic CT. $J$ Vasc Interv Radiol 2006;17:1349-53.

12. Georgiades CS, Hong K, Geschwind JF, Liddell R, Syed L, Kharlip J, Arepally A. Adjunctive use of C-arm CT may eliminate technical failure in adrenal vein sampling. J Vasc Interv Radiol 2007;18:1102-5.

13. Virmani S, Ryu RK, Sato KT, Lewandowski RJ, Kulik L, Mulcahy MF, Larson AC, Salem R, Omary RA. Effect of C-arm angiographic CT on transcatheter arterial chemoembolization of liver tumors. $J$ Vasc Interv
Radiol 2007;18:1305-9.

14. Becker HC, Meissner O, Waggershauser T. C-arm CT-guided 3D navigation of percutaneous interventions. Radiologe 2009;49:852-5. (in German)

15. Miyayama S, Yamashiro M, Okuda M, Yoshie Y, Sugimori N, Igarashi S, Nakashima Y, Matsui O. Usefulness of cone-beam computed tomography during ultraselective transcatheter arterial chemoembolization for small hepatocellular carcinomas that cannot be demonstrated on angiography. Cardiovasc Intervent Radiol 2009;32:255-64

16. Kakeda S, Korogi Y, Ohnari N, Moriya J, Oda N, Nishino K, Miyamoto W. Usefulness of cone-beam volume CT with flat panel detectors in conjunction with catheter angiography for transcatheter arterial embolization. J Vasc Interv Radiol 2007;18:1508-16.

17. Golfieri R, Renzulli M, Lucidi V, Corcioni B, Trevisani F, Bolondi L. Contribution of the hepatobiliary phase of Gd-EOB-DTPA-enhanced MRI to Dynamic MRI in the detection of hypovascular small $(\leq 2 \mathrm{~cm})$ HCC in cirrhosis. Eur Radiol 2011;21:1233-42.

18. Furuta T, Maeda E, Akai H, Hanaoka S, Yoshioka N, Akahane M, Watadani T, Ohtomo K. Hepatic segments and vasculature: projecting CT anatomy onto angiograms. Radiographics 2009;29:1-22.

19. Saylisoy S, Atasoy C, Ersöz S, Karayalçin K, Akyar S. Multislice CT angiography in the evaluation of hepatic vascular anatomy in potential right lobe donors. Diagn Interv Radiol 2005;11:51-9.

20. Kim HC, Chung JW, Jae HJ, Yoon JH, Lee JH, Kim YJ, Lee HS, Yoon CJ, Park JH. Caudate lobe hepatocellular carcinoma treated with selective chemoembolization. Radiology 2010;257: 278-87.

21. Miyayama S, Yamashiro M, Yoshie Y, Nakashima Y, Ikeno H, Orito $\mathrm{N}$, Yoshida M, Matsui O. Hepatocellular carcinoma in the caudate lobe of the liver: variations of its feeding branches on arteriography. Jpn J Radiol 2010;28:555-62.

22. Abdalla EK, Vauthey JN, Couinaud C. The caudate lobe of the liver: implications of embryology and anatomy for surgery. Surg Oncol Clin N Am 2002;11:835-48.

23. Wallace MJ. C-arm computed tomography for guiding hepatic vascular interventions. Tech Vasc Interv Radiol 2007;10:79-86. 